\title{
Electron-Mediated Nuclear-Spin Interactions between Distant Nitrogen-Vacancy Centers
}

\author{
A. Bermudez, ${ }^{1}$ F. Jelezko, ${ }^{2}$ M. B. Plenio, ${ }^{1}$ and A. Retzker ${ }^{1}$ \\ ${ }^{1}$ Institut für Theoretische Physik, Albert-Einstein Allee 11, Universität Ulm, 89069 Ulm, Germany \\ ${ }^{2}$ Institut für Quantenoptik, Albert-Einstein Allee 11, Universität Ulm, 89069 Ulm, Germany
}

(Received 25 July 2011; published 3 October 2011)

\begin{abstract}
We propose a scheme enabling controlled quantum coherent interactions between separated nitrogenvacancy centers in diamond in the presence of strong magnetic fluctuations. The proposed scheme couples nuclear qubits employing the magnetic dipole-dipole interaction between the electron spins and, crucially, benefits from the suppression of the effect of environmental magnetic field fluctuations thanks to a strong microwave driving. This scheme provides a basic building block for a full-scale quantum-information processor or quantum simulator based on solid-state technology.
\end{abstract}

DOI: 10.1103/PhysRevLett.107.150503

The spins of single dopants in solids are key elements in the development of solid-state quantum-information technologies [1,2]. In particular, nitrogen-vacancy (NV) color centers in diamond are promising quantum processors: single defects can be detected using confocal microscopy $[3,4]$, their spin state can be initialized, manipulated, and readout optically [5-8], and their quantum coherence survives at room temperatures [9]. One of the remaining challenges is to control the spin-spin interactions to perform quantum-logic operations, and major steps along this direction have already been accomplished. The hyperfine coupling between the NV electron spin and the nuclear spins of neighboring impurities $\left({ }^{13} \mathrm{C},{ }^{15} \mathrm{~N}\right)$ offers a unique opportunity to build small quantum registers [8,10-13]. These devices can be scaled up by means of ion implantation techniques, yielding periodic arrays of NV centers [14]. However, the controlled couplings now require longer-range interactions, as provided by optical channels [15], or magnetic dipole-dipole couplings between the electron spins [16].

Although the feasibility of the magnetic-coupling approach has been demonstrated recently [16], fabricated NV arrays often suffer from shorter electron coherence times that affect the fidelity of the quantum gates. From this perspective, ${ }^{14} \mathrm{~N}$ or ${ }^{15} \mathrm{~N}$ nuclear spins would be bettersuited qubits due to their longer coherence times, together with the availability of single-shot readout [10]. Unfortunately, the direct nuclear dipole-dipole interaction is negligible, which necessitates the search for alternative schemes to couple the nuclear spins. This Letter presents a theoretical proposal for implementing robust quantum gates between two distant nuclear-spin qubits mediated by the long-range dipolar interaction between electron spins. The main idea is to exploit the long nuclear coherence times for storage and to use the electronic degrees of freedom as a quantum bus that mediates the nuclear-spin interaction. Such a general scheme can be applied to different setups, and has also been proposed for quantum-Hall systems [17]. Active control of the spins via microwave fields allows reaching high fidelities, even in the presence
PACS numbers: 03.67.Lx, 03.67.Pp, 74.78.Na, 76.30.Mi

of the magnetic noise associated with the complex mesoscopic environment of solid-state systems. In fact, the nuclear driving acts as a continuous decoupling mechanism [18] that minimizes the effects of the noise and provides a new tool in addition to pulsed techniques $[19,20]$.

Model.-We consider two NV defects $j=1,2$, whose unpaired electrons form a spin-triplet ground state $S_{j}=1$ and focus on ${ }^{14} \mathrm{~N}$ with a nuclear spin $I_{j}=1$. The Hamiltonian that describes each NV center is $H_{j}=H_{j}^{(e)}+$ $H_{j}^{(n)}+H_{j}^{(e-n)}$,

$$
\begin{aligned}
H_{j}^{(e)} & =D_{j}\left(\left(S_{j}^{z}\right)^{2}-\frac{1}{3} \boldsymbol{S}_{j}^{2}\right)+g_{e} \mu_{B} \boldsymbol{B} \cdot \boldsymbol{S}_{j}, \\
H_{j}^{(n)} & =-P_{j}\left(\left(I_{j}^{z}\right)^{2}-\frac{1}{3} \boldsymbol{I}_{j}^{2}\right)-g_{n} \mu_{N} \boldsymbol{B} \cdot \boldsymbol{I}_{j}, \\
H_{j}^{(e-n)} & =A_{j}^{\|} S_{j}^{z} I_{j}^{z}+\frac{1}{2} A_{j}^{\perp}\left(S_{j}^{+} I_{j}^{-}+S_{j}^{-} I_{j}^{+}\right),
\end{aligned}
$$

where $\boldsymbol{S}_{j}, \boldsymbol{I}_{j}$ are the electronic and nuclear spin-1 operators, and $S_{j}^{ \pm}=S_{j}^{x} \pm i S_{j}^{y}, I_{j}^{ \pm}=I_{j}^{x} \pm i I_{j}^{y}$ the usual ladder operators. Here, $D_{j}\left(P_{j}\right)$ stands for the zero-field splitting of the electronic (nuclear) ground state, $\boldsymbol{B}$ is an external magnetic field, $\mu_{B}\left(\mu_{N}\right)$ is the Bohr (nuclear) magneton, and $g_{e}\left(g_{n}\right)$ is the electron (nuclear) $g$ factor. The electronnuclei interaction is quantified by the hyperfine longitudinal (transverse) coupling $A_{j}^{\|}\left(A_{j}^{\perp}\right)$. The present discussion is focused on a single pair of closely spaced NV centers, and we use the realistic parameters of the experiment in [16]. We emphasize, however, that this scheme can be extended to arrays of implanted NV centers, provided that their distance is small enough. Note that the hierarchy in the coupling strengths, $D_{j} \gg P_{j} \geq A_{j}^{\|}, A_{j}^{\perp}$ and $g_{e} \mu_{B} \gg$ $g_{n} \mu_{N}$, shall play a crucial role in our scheme (see Table I, where $\hbar=1)$. Finally, we introduce the secular dipoledipole interaction between the electron spins

$$
H_{12}^{(e-e)}=J_{12}\left(3 S_{1}^{z} S_{2}^{z}-S_{1} \cdot S_{2}\right),
$$


TABLE I. Specific values of the coupling strengths.

\begin{tabular}{lcccccccccc}
\hline \hline$D_{j}$ & $P_{j}$ & $A_{j}^{\|}, A_{j}^{\perp}$ & $J_{12}$ & $g_{e} \mu_{B}$ & $g_{n} \mu_{N}$ & $B$ & $\Omega_{e}$ & $\Omega_{n}$ & $J_{\text {eff }}^{x x}$ & $J_{\text {eff }}^{z z}$ \\
\hline $2.87 \mathrm{GHz}$ & $5.04 \mathrm{MHz}$ & $2.1,2.3 \mathrm{MHz}$ & $70 \mathrm{kHz}$ & $2.8 \mathrm{MHz} \cdot \mathrm{G}^{-1}$ & $0.31 \mathrm{kHz} \cdot \mathrm{G}^{-1}$ & $30 \mathrm{G}$ & $15 \mathrm{MHz}$ & $1 \mathrm{kHz}$ & $0.1 \mathrm{~Hz}$ & $0.1 \mathrm{kHz}$ \\
\hline \hline
\end{tabular}

where $J_{12}=g_{e}^{2} \mu_{B}^{2}\left(1-3 \cos ^{2} \theta_{12}\right) / 2 c r_{12}^{3}$ in Gaussian units, $\boldsymbol{r}_{12}$ is the distance between the NV centers, $\cos \theta_{12}=\boldsymbol{e}_{z}$. $\boldsymbol{r}_{12} / r_{12}$, and $c$ is the speed of light. For the distances reached in the experiment, $r_{12} \approx 10 \mathrm{~nm}$, the dipolar coupling $J_{12} \approx 70 \mathrm{kHz}$ is the smaller energy scale in the problem. As mentioned above, the magnetic dipole-dipole interaction between the nuclear spins is completely negligible since $\left(g_{n} \mu_{N} / g_{e} \mu_{B}\right)^{2} \approx 10^{-8}$, and an indirect mechanism for the nuclear coupling is thus required.

Effective static interactions.-In Fig. 1(a), we represent schematically the process leading to nuclear spin-spin interactions. The hyperfine interaction couples the nuclear to the electronic spins of each NV center, which are in turn coupled through the magnetic dipole-dipole interaction. Therefore, one may use the electrons as a bus to mediate the nuclear coupling. A naive estimate of this coupling follows from Fig. 1(b), where we represent the energy spectrum of $H_{0}=\sum_{j}\left(H_{j}^{(e)}+H_{j}^{(n)}\right)+H_{12}^{(e-e)}$. Because of the energy-scale hierarchy in Table I, the levels are clustered in manifolds determined by the electronic spins $\left|m_{1}, m_{2}\right\rangle_{e}$. The dynamics within the ground-state manifold, $|0,0\rangle_{e}$, corresponds to nuclear-spin flips $\left|M_{1}, M_{2}\right\rangle_{n} \rightarrow\left|M_{1}^{\prime}, M_{2}^{\prime}\right\rangle_{n}$, with $M_{j}, M_{j}^{\prime}=0, \pm 1$, and follows from second-order processes where the hyperfine coupling virtually populates states from the excited manifold. Therefore, a crude estimate of the dynamics is $H_{\text {eff }} \approx J_{\text {eff }} I_{1}^{+} I_{2}^{-}+$H.c., where $J_{\text {eff }} \propto\left(A_{1}^{\perp} A_{2}^{\perp}\right) / D$. A more careful Schrieffer-Wolff-type calculation takes into account the two possible channels [21], symmetric or antisymmetric, which lead to the destructive interference of this coupling $J_{\text {eff }} \propto\left(A_{1}^{\perp} A_{2}^{\perp}\right) / D-$ $\left(A_{1}^{\perp} A_{2}^{\perp}\right) / D$. It is precisely the role of the magnetic dipoledipole interaction to split these channels, suppressing the perfect destructive interference, and leading to

$$
\begin{aligned}
& H_{\text {eff }}^{x x}=J_{\text {eff }}^{x x}\left(I_{1}^{+} I_{2}^{-}+I_{1}^{-} I_{2}^{+}\right)-\sum_{j} P_{j}\left(I_{j}^{z}\right)^{2}, \\
& J_{\text {eff }}^{x x}=\frac{2 A_{1}^{\perp} A_{2}^{\perp}}{D^{2}} J_{12} .
\end{aligned}
$$
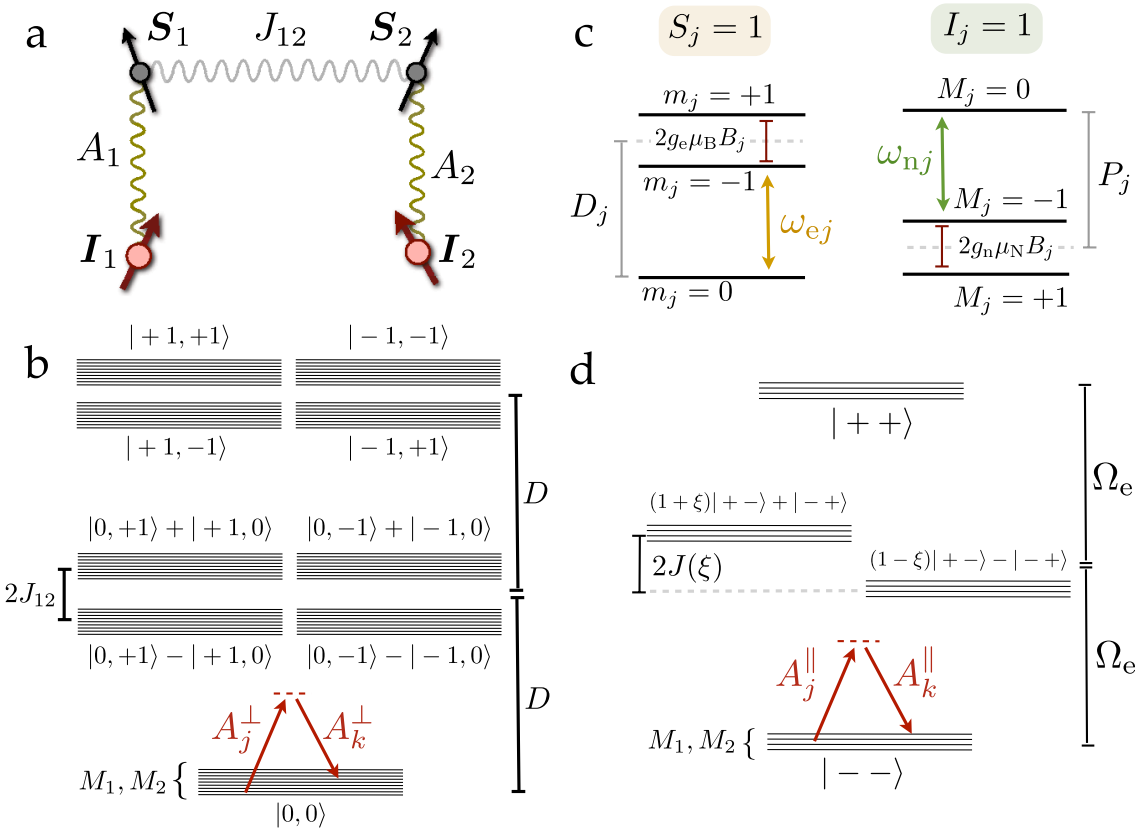

d

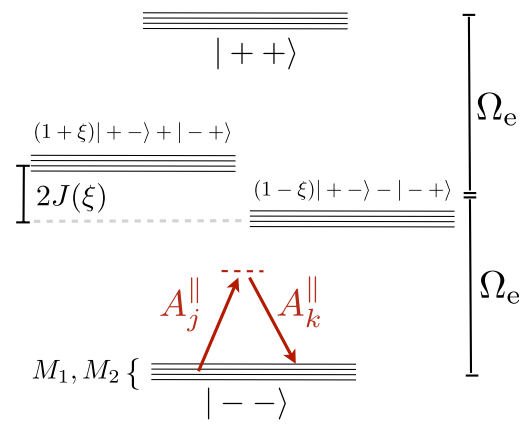

FIG. 1 (color online). Effective nuclear spin-spin interaction. (a) Schematic diagram of the electron-mediated interaction between the nuclear spins, which exploits the magnetic dipolar interaction and the local hyperfine coupling. (b) Diagram of the energy levels of the two NV centers. Since $D_{j}$ is the largest energy scale, the energies are clustered in manifolds determined by the electronic spin. The transverse part of the hyperfine coupling $A_{j}^{\perp}$ induces transitions between different manifolds and mediates an effective $X X$ interaction between the nuclear spins. (c) Schematic diagram of the Zeeman splitting for the electronic and nuclear energy levels. By carefully selecting the microwave frequencies, we drive a particular electronic and nuclear transition. (d) Energy levels of the driven Hamiltonian. For very strong driving $\Omega_{e}$, the electronic spin in the lowest manifold is $|-\rangle \propto|0\rangle-|-1\rangle$. The hyperfine coupling $A_{j}^{\|}$induces virtual transitions to the excited manifold, split by the dipolar interaction and the inhomogeneous broadening, and leads to an effective $Z Z$ interaction. 
This Hamiltonian describes the flip-flop interaction between the ${ }^{14} \mathrm{~N}$ nuclei leading to an exchange of the spin excitations.

In Fig. 2(a), we present a scheme for the electronmediated gate between two NV nuclei based on Eq. (3), referred to as the nuclear $X X$ gate. The initialization yields the state $\left|\psi_{0}\right\rangle=\left|\phi_{e}\right\rangle \otimes\left|\varphi_{n}\right\rangle=|0,0\rangle_{e} \otimes|0,1\rangle_{n}$, where electrons belong to the ground-state manifold of Fig. 1(b), and the dynamics of the spin excitation is determined by virtual electron spin-flip processes. In Fig. 2(c), we study numerically the accuracy of the effective Hamiltonian (3), which is compared to the exact evolution under the total Hamiltonian (1) and (2). One observes that the electron state remains in the ground state, whereas there is a periodic exchange of the spin excitation between the nuclei. The remarkable agreement of both predictions justifies the validity of the effective nuclear spin-spin Hamiltonian in Eq. (3). Unfortunately, the parameters in Table I yield a vanishingly small coupling $J_{\text {eff }}^{x x} \approx 0.1 \mathrm{~Hz}$, which is far too slow to produce any observable coherent coupling between the nuclei. Even if not of practical use, the above derivation gives a neat account of the mechanism of electron-mediated interactions, and will help us in understanding how to raise the interaction strength.
A possibility to overcome this problem is to apply a magnetic field, such that the Zeeman shift reduces $D \rightarrow$ $D-g_{e} \mu_{B} B$, thus enhancing $J_{\text {eff }}^{x x}$. Yet, one faces two important problems. (i) In general, the axes of the NV centers are not aligned, and each electronic spin experiences a different Zeeman shift. For the large fields required, this inhomogeneity might exceed the dipolar coupling, and thus spoil the scheme. (ii) The dephasing exerted by the environment would have a contribution that ruins the coherence of the interaction. We demonstrate below that there is a different approach that overcomes both problems simultaneously, and yet enhances the nuclear-spin interaction: continuous microwave driving [18].

Effective driven interactions. - We now discuss the effects of a continuous microwave field that drives both the electronic and nuclear spins. The effect of the driving is twofold. (i) By addressing each NV center with different microwave fields, one can independently tune their frequencies so that they become resonant with a particular transition. This allows us to overcome the problems associated with both the inhomogeneous broadening and the different Zeeman shifts. Moreover, this can be used for single addressing of NVs, especially when combined with magnetic gradients. (ii) By tuning the microwave a

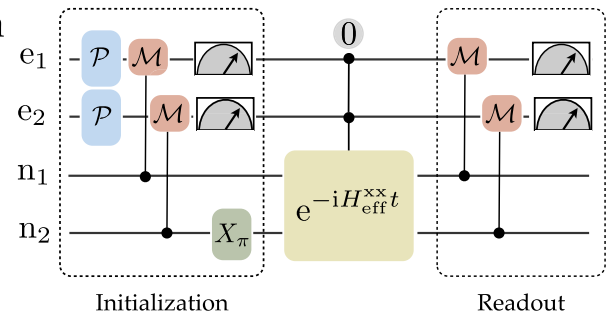

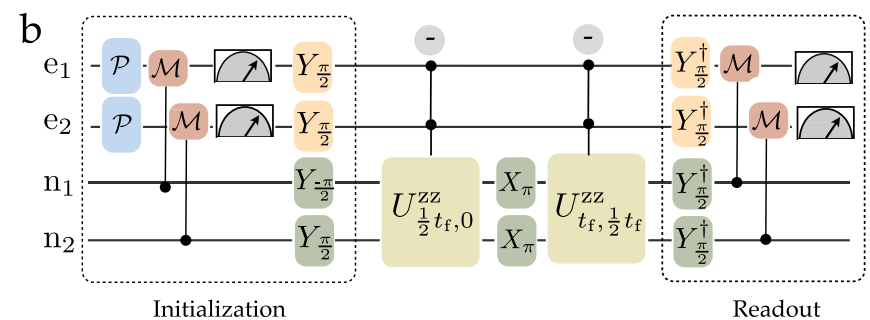

Initialization
Readout

$\mathrm{e}$

C $\mathrm{d}$

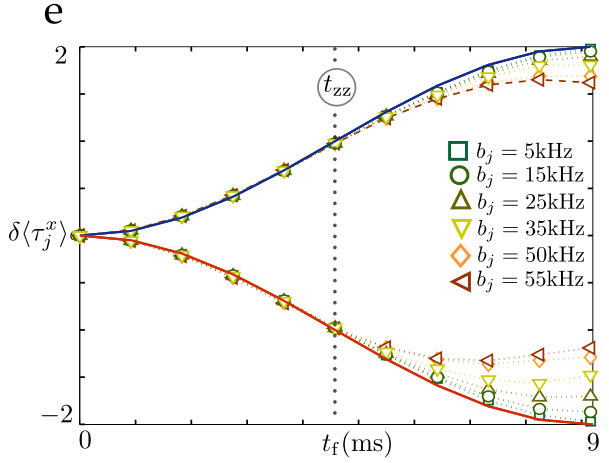

FIG. 2 (color online). Nuclear quantum gate between two NV centers. Scheme for the initialization, evolution, and readout of the effective $J_{\text {eff }}^{x x}$ interaction in (a), and $J_{\text {eff }}^{z z}$ interaction in (b). Here, $X_{j, \phi}=\exp \left(i \phi \tau_{j}^{x} / 2\right), Y_{j, \theta}=\exp \left(i \theta \tau_{j}^{y} / 2\right)$ represent finite pulses, and $\mathcal{P}$ $(\mathcal{M})$ stand for the electron (nuclear) spin polarization. (c) Comparison between the effective dynamics under the Hamiltonian (3) and the exact time evolution under Eqs. (1) and (2) for the $J_{\text {eff }}^{x x}$ nuclear interaction. The expectation values represented correspond to the nuclear spin $\left\langle I_{j}^{z}\right\rangle$ and electronic spin $\left\langle S_{\text {tot }}^{z}\right\rangle=\left\langle S_{1}^{z}+S_{2}^{z}\right\rangle$. (d) Comparison between the exact (5) and effective (6) dynamics for the $J_{\text {eff }}^{z z}$ nuclear interaction, together with an echo scheme that allows us to get rid of the fast single-nuclei dynamics. We represent the nuclear expectation values $\delta\left\langle\tau_{j}^{x}\right\rangle=\left\langle\tau_{j}^{x}\right\rangle_{t_{f}}-\left\langle\tau_{j}^{x}\right\rangle_{t_{0}}$. The dotted lines correspond to $J_{12}=0$, where there is no interaction induced on the nuclei. (e) Performance of the $Z Z$ gate in the presence of different strengths of the electron dephasing noise $b_{j}=$ $\{5,15,25,35,50,55\} \mathrm{kHz}$, where the nuclear noise is $B_{j}=0.1 b_{j}$. The corresponding Ramsey decoherence times are roughly $T_{2 e} \approx\{0.2,0.07,0.04,0.03,0.02,0.018\} \mathrm{ms}, T_{2 n}=0.1 T_{2 e}$. 
frequency on resonance with the transition, one introduces a new energy scale that governs the system, namely, the Rabi frequency. This parameter can be tuned by controlling the microwave power, allowing us to enhance $J_{\text {eff }}$.

Let us consider the Zeeman effect associated with $B=$ $30 \mathrm{G}$ in Fig. 1(c). By setting the microwave frequencies to $\omega_{e j}=D_{j}-g_{e} \mu_{B} B_{j}, \quad \omega_{n j}=P_{j}-g_{n} \mu_{N} B_{j}$, one resonantly drives the transitions between the electronic and nuclear levels $m_{j}=0 \leftrightarrow-1, M_{j}=0 \leftrightarrow-1$. These driving terms can be written as

$$
H_{d}(t)=\sum_{j} \Omega_{e} \sigma_{j}^{x} \cos \omega_{e_{j}} t+\Omega_{n} \tau_{j}^{x} \cos \omega_{n_{j}} t,
$$

where the Rabi frequencies of the electronic and nuclear transitions are $\Omega_{e}, \Omega_{n}$, and the electronic and nuclear Pauli matrices $\sigma_{j}^{x}, \tau_{j}^{x}$. In the interaction picture with respect to $H_{0,1}=\sum_{j} D_{j}\left(S_{j}^{z}\right)^{2}-P_{j}\left(I_{j}^{z}\right)^{2}+g_{e} \mu_{B} B_{j} S_{j}^{z}-g_{n} \mu_{N} B_{j} I_{j}^{z}$, one can neglect the rapidly oscillating terms associated with the transverse part of the Zeeman shifts and the hyperfine coupling. This rotating wave approximation is justified for the parameters shown in Table I. Additionally, we consider two NV centers with different axes, which allows us to neglect the transverse part of the magnetic dipole coupling. For weak-enough driving, we arrive at the total driven Hamiltonian

$$
\begin{aligned}
& H_{0}=\sum_{j}\left(\frac{1}{2} \Omega_{e} \sigma_{j}^{x}+\frac{1}{2} \Omega_{n} \tau_{j}^{x}\right)+2 J_{12} S_{1}^{z} S_{2}^{z}, \\
& H_{1}=\sum_{j} A_{j}^{\|} S_{j}^{z} I_{j}^{z} .
\end{aligned}
$$

We stress that these approximations are justified by the parameters in Table I and supported by numerical simulations.

We now derive the electron-mediated nuclear-spin interactions starting from Eq. (5). We note that there is again a hierarchy in the couplings $\Omega_{e} \gg A_{j}^{\|} \gg J_{12} \gg \Omega_{n}$, which leads to the clustering of energy levels shown in Fig. 1(d). By considering the electron ground state, the nuclear spins can interact through virtual electron spin flips to the excited manifolds. In this driven regime, it is the longitudinal hyperfine coupling $A_{j}^{\|}$that induces such virtual transitions. A Schrieffer-Wolff-type calculation yields the nuclear Hamiltonian

$$
\begin{aligned}
& H_{\mathrm{eff}}^{z z}=J_{\mathrm{eff}}^{z z} \tau_{1}^{z} \tau_{2}^{z}+\sum_{j} \Omega_{n} \tau_{j}^{x}-\frac{1}{4} A_{j}^{\|} \tau_{j}^{z}, \\
& J_{\mathrm{eff}}^{z z}=\frac{-A_{1}^{\|} A_{2}^{\|}}{8 \Omega_{e}}\left(\frac{J_{12}}{\Omega_{e}}+2 \xi\right),
\end{aligned}
$$

where we considered the inhomogeneous broadening of the hyperfine couplings $\xi=2\left[\left(A_{2}^{\|}\right)^{2}-\left(A_{1}^{\|}\right)^{2}\right] / \Omega_{e} J_{12}$. This Hamiltonian is an Ising magnetic interaction between the nuclear spins, which are additionally subjected to a transverse field due to the driving and a longitudinal field due to the hyperfine coupling. As advanced previously, we have been able to enhance the electron-mediated nuclear interaction, which becomes $J_{\text {eff }}^{z z} \approx 0.1 \mathrm{kHz}$ for the parameters in Table I. Remarkably, the strength of the nuclear-spin interaction has increased by 3 orders of magnitude $J_{\text {eff }}^{z z} \approx$ $10^{3} J_{\text {eff }}^{x x}$.

In Fig. 2(b), we schematically describe the necessary ingredients for the nuclear $Z Z$ gate. The initialization consists of the electron (nuclear) spin polarization $\mathcal{P}$ $(\mathcal{M})$, together with single-spin gates. $\mathcal{P}$ is obtained by the optical pumping cycle available for NV centers $[5,6]$, whereas $\mathcal{M}$ is based on the techniques developed for the nuclear single-shot measurement [10], followed by the electron state-dependent fluorescence $[5,6]$. Once polarized, $|0,0\rangle_{e} \otimes|0,0\rangle_{n}$, one applies unitary gates based on microwave pulses of different duration, $Y_{j, \pi / 2}=\left(\square+i \tau_{j}^{y}\right)$, $Y_{j,-\pi / 2}=\left(\square-i \tau_{j}^{y}\right)$ (also for the electron spin), which lead to $\left|\psi_{0}\right\rangle=|--\rangle_{e} \otimes|-+\rangle_{n}$. The evolution of this state is dictated by the interaction picture Hamiltonian (6), which leads to $U_{t_{2}, t_{1}}^{z z}=e^{-i H_{0,1} t_{2}} e^{-i H_{\mathrm{eff}}^{z z}\left(t_{2}-t_{1}\right)} e^{+i H_{0,1} t_{1}}$. Because of the longitudinal field, and the additional contributions of $H_{0,1}$, the simple periodic exchange of the nuclear-spin excitation will be accompanied by fast oscillations. In order to observe neatly the effect of the interaction, one may perform a spin-echo sequence, such that the nuclear spins are inverted at half the gate time by a microwave pulse $X_{j, \pi}=i \tau_{j}^{x}$. In this case, the fast single-nuclei oscillations refocus after the spin-echo period $t_{f}$, and one observes solely the effect of the interaction. In Fig. 2(d), we compare the effective description (6) to the Hamiltonian (5), which displays a clear agreement. In particular, when the echo period matches twice the $Z Z$-gate time $t_{f}=$ $2 t_{z z}=\pi / 2 J_{\text {eff }}^{z z} \approx 9 \mathrm{~ms}$, one finds a perfect excitation exchange $\left\langle\tau_{1}^{x}\right\rangle:-1 \rightarrow+1,\left\langle\tau_{2}^{x}\right\rangle:+1 \rightarrow-1$. Note that for $J_{12}=0$, this effect is completely absent. Finally, considering $t_{f}=t_{z z}$, and setting the echo pulse along the $y$ axis, the dynamics generates an entangled nuclear state $\left|\psi_{0}\right\rangle=$ $|--\rangle_{e} \otimes\left|-{ }_{y}+_{y}\right\rangle_{n} \rightarrow\left|\psi_{f}\right\rangle=|--\rangle_{e} \otimes\left(\left|-{ }_{y}+_{y}\right\rangle_{n}+\right.$ $\left.\left|+{ }_{y}-\right\rangle_{n}\right) / \sqrt{2}$. Once the gate has been performed, the nuclear operators $\left\langle I_{j}^{z}\right\rangle,\left\langle\tau_{j}^{x}\right\rangle$ must be measured. Since the state-dependent fluorescence is particular to the electron spins, one should map the nuclear information onto the electrons, and then measure. This can be achieved in a quantum nondemolition fashion by using a microwave on an electron-spin transition conditioned to the nuclei [10].

Decoupling from decoherence.-So far, our discussion has focused on the idealized situation of isolated NV centers. However, every quantum system is inevitably coupled to an environment that degrades its coherence. This phenomenon, known as decoherence, must be seriously accounted for in solid-state materials, where the system-environment coupling is usually strong. In the particular case of $\mathrm{NV}$ centers, the major source of decoherence is the coupling to other impurity spins, such as single substitutional nitrogen electron spin (P1 center) in 
type $\mathrm{Ib}$ diamond [22], or ${ }^{13} \mathrm{C}$ isotopes in type IIa [8]. The microscopic description of the spin bath is an intricate many-body problem, and is a current subject of intense research. Here, we use a phenomenological model of the bath that yields a fluctuating magnetic field shifting the resonance frequencies. Because of the spin interactions, this effective field is modeled as a stochastic OrnsteinUhlenbeck process [19,23]

$$
H_{\text {noise }}=\sum_{j}\left(b_{j}(t) S_{j}^{z}+B_{j}(t) I_{j}^{z}\right),
$$

where $b_{j}(t), B_{j}(t)$ are random processes with autocorrelation $\left\langle b_{j}(t) b_{j}(0)\right\rangle=b_{j}^{2} e^{-r_{j} t},\left\langle B_{j}(t) B_{j}(0)\right\rangle=B_{j}^{2} e^{-R_{j} t}$, where $b_{j}^{2}, B_{j}^{2}$ represent variance of the zero-mean Gaussian distributions, and $r_{j}, R_{j}$ the inverse of their correlation times. In particular, the decoherence time of an electronic (nuclear) Ramsey experiment is given by $T_{2 e}=1 / b_{j}$ $\left(T_{2 n}=1 / B_{j}\right)$. By considering the particular time dependence of these stochastic processes, we numerically integrate the noisy dynamics and average for $N=10^{3}$ realizations of the random process. This allows us to study the effects of decoherence on the gate.

For the slow $X X$ gate [Fig. 2(a)], the limiting factor is the nuclear dephasing time, which can attain values of $T_{2 n} \approx 10 \mathrm{~ms}$. Even for the purest samples, the coherence of the gate is completely lost long before the target time $t_{x x} \approx 4.5 \mathrm{~s}$ is reached. Therefore, the performance of this gate is extremely poor. For the fast $Z Z$ gate [Fig. 2(b)], not only the nuclear-spin dephasing but also the electron-spin dephasing limit the gate accuracy. In the dressed-state basis [see Fig. 1(d)], the electron dephasing tries to induce a transition between the different manifolds, introducing additional noise in the nuclei. However, due to the strong driving $\Omega_{e}$, these processes are partially suppressed. Additionally, a sufficiently strong nuclear driving, $\Omega_{n} \gg$ $B_{j}\left(b_{j} A_{j}^{\|} / \Omega_{e}\right)$, provides an additional decoupling mechanism that further enhances the gate performance. In Fig. 2(e), one observes the announced decoupling, since the gate performance at the target time $t_{z z} \approx 4.5 \mathrm{~ms}$ is extremely good even for shorter electronic coherence times ranging from $T_{2 e} \approx 0.1 \mathrm{~ms}$ to $T_{2 e} \approx 50 \mu \mathrm{s}$. Because of the decoupling mechanism, the gate accuracy will actually be limited by the decay times $T_{1 e}$. Moreover, at this time scale, energy will be pumped into the system by the continuous driving. However, note that this limitation can be overcome since $T_{1 e}$ can be increased by orders of magnitude by cooling. Accordingly, one can achieve high fidelities.

Let us finally note that the effective decoupling mechanism presented here can also be used to improve the electron-spin gates based on the direct dipole interaction [16]. In that case, the role of the microwave driving is to prolong dephasing times and to bring the two dressed electronic transitions to resonance to overcome the inhomogeneous broadening.
Conclusions and outlook.-We have demonstrated the feasibility for engineering electron-mediated spin-spin interactions between the nuclei of two NV centers. By continuous microwave driving, this scheme allow us to decouple from the electronic and nuclear dephasing sources, and increase the effective interactions by 3 orders of magnitude, thus achieving $J_{\text {eff }}^{z z} \approx 0.1 \mathrm{kHz}$ for distances of existing pairs of NV centers [16]. This scheme opens the possibility for the realization of quantum-information processors, quantum simulators, and quantum sensors [24] on the basis of NV centers in diamond. Finally, we would like to stress the generality of this scheme, which can be applied to other solid-state technologies that are candidates for quantum-information processing.

This work was supported by the EU STREP projects HIP, PICC, and by the Alexander von Humboldt Foundation. We thank J. Cai for useful discussions.

[1] J. R. Weber et al., Proc. Natl. Acad. Sci. U.S.A. 107, 8513 (2010).

[2] T. D. Ladd et al., Nature (London) 464, 45 (2010).

[3] A. Gruber et al., Science 276, 2012 (1997).

[4] C. Kurtsiefer et al., Phys. Rev. Lett. 85, 290 (2000).

[5] F. Jelezko et al., Appl. Phys. Lett. 81, 2160 (2002).

[6] F. Jelezko et al., Phys. Rev. Lett. 92, 076401 (2004).

[7] R. J. Epstein et al., Nature Phys. 1, 94 (2005).

[8] L. Childress et al., Science 314, 281 (2006).

[9] G. Balasubramanian et al., Nature Mater. 8, 383 (2009).

[10] P. Neumann et al., Science 329, 542 (2010).

[11] T. Gaebel et al., Nature Phys. 2, 408 (2006).

[12] P. Neumann et al., Science 320, 1326 (2008).

[13] M. V. Gudurev Dutt et al., Science 316, 1312 (2007).

[14] J. Meijer et al., Appl. Phys. A 83, 321 (2006); W. Schnitzler et al., Phys. Rev. Lett. 102, 070501 (2009); D. M. Toyli et al., Nano Lett. 10, 3168 (2010).

[15] E. Togan et al., Nature (London) 466, 730 (2010); Y. Kubo et al., Phys. Rev. Lett. 105, 140502 (2010); A. D. Greentree et al., J. Phys. Condens. Matter 18, S825 (2006).

[16] P. Neumann et al., Nature Phys. 6, 249 (2010).

[17] V. Privman, I. D. Vagner, and G. Kventsel, Phys. Lett. A 239, 141 (1998).

[18] N. Timoney et al., Nature (London) 476, 185 (2011); B. Naydenov et al., Phys. Rev. B 83, 081201 (2011).

[19] G. de Lange et al., Science 330, 60 (2010).

[20] H. Bluhm et al., Nature Phys. 7, 109 (2010).

[21] See Supplemental Material at http://link.aps.org/ supplemental/10.1103/PhysRevLett.107.150503 for a detailed discussion of the effective nuclear-spin interactions, and the phenomenological noise model.

[22] R. Hanson et al., Science 320, 352 (2008).

[23] V. V. Dobrovitski et al., Phys. Rev. Lett. 102, 237601 (2009).

[24] G. Balasubramanian et al., Nature (London) 455, 648 (2008); J. M. Taylor et al., Nature Phys. 4, 810 (2008); J. R. Maze et al., Nature (London) 455, 644 (2008); L. T. Hall et al., Proc. Natl. Acad. Sci. U.S.A. 107, 18777 (2010). 